\title{
Heavy ion storage ring without linear dispersion
}

\author{
Masahiro Ikegami, Akira Noda, and Mikio Tanabe \\ Institute for Chemical Research, Kyoto University, Gokanosho, Uji, 611-0011, Kyoto, Japan \\ Manfred Grieser \\ Max-Planck-Institut für Kernphysik, Postfach 103980, 69029 Heidelberg, Germany
}

Hiromi Okamoto

Graduate School of Advanced Sciences of Matter, Hiroshima University,

1-3-1 Kagamiyama, Higashi-Hiroshima, 739-8530, Hiroshima, Japan

(Received 4 April 2003; revised manuscript received 13 October 2004; published 16 December 2004)

\begin{abstract}
A possible method to realize a dispersion-free storage ring is described. The simultaneous use of a magnetic field $\mathbf{B}$ and an electric field $\mathbf{E}$ in bending regions, where the two fields are set perpendicular to each other, enables us to control the effect of momentum dispersion. When the relation $\left(1+1 / \gamma_{0}^{2}\right) \mathbf{E}(\rho)=$ $-\mathbf{v}_{0} \times \mathbf{B}$ is satisfied for a beam with the velocity $v_{0}$, the linear dispersion can be completely eliminated all around the ring. It is shown that the acceleration and deceleration induced by the electrostatic deflector counteracts the heating mechanism due to the shearing force from dipole magnets. The dispersion-free system is thus beneficial to producing ultracold beams. It looks probable that the technique will allow one to achieve three-dimensional crystalline beams. At ICR Kyoto University, an ion cooler storage ring SLSR oriented for various beam physics purposes is now under construction. The application of the present idea to S-LSR is discussed and the actual design of the dispersionless bend is given.
\end{abstract}

DOI: 10.1103/PhysRevSTAB.7.120101

PACS numbers: 41.85.Lc, 29.20.Dh, 41.75.-i, 52.65.Cc

\section{INTRODUCTION}

In recent years, considerable experimental effort has been devoted in various fields to realize a low temperature state of a multiparticle system. Laser cooling is the most powerful means for this purpose. It is often applied to charged particles confined in a plasma trap where Coulomb crystallization, an ultimate low temperature state, has already been achieved [1-3]. In the field of beam physics, other cooling techniques, such as electron cooling and stochastic cooling, have also been employed to control the rest-frame temperature of ion beams. By using the electron cooling technique, one-dimensional ordering of an ultra-low-density ion beam has been established at ESR and CRYRING [4,5] (although it is physically different from a crystalline state [6]). The laser cooling experiment at PALLAS, a circular RFQ trap [7], has shown the observation of 2D and 3D crystalline beams at the very low beam energy around $1 \mathrm{eV}$. Furthermore, the realization of a bunched crystal beam has been reported [8]. However, the 3D crystallization of "fast ion beams" has not been accomplished in storage rings yet.

Noting the similarity between ion traps and storage rings, we naturally expect that Coulomb crystallization may be achievable even for a fast stored beam. Molecular dynamics (MD) studies have actually demonstrated the possibility of a phase transition to a crystalline beam $[9,10]$. According to advanced MD simulations where the realistic lattice structure of a storage ring is incorporated, a variety of crystalline configurations can be formed in a properly designed ring when a sufficiently strong three-dimensional (3D) cooling force is available [10-13]. On the other hand, it has also been pointed out that the stability of a multidimensional crystalline beam can be seriously affected by a dynamic effect peculiar to storage rings.

The most essential difference between ion traps and storage rings is whether the effect of momentum dispersion exists. In circular machines such as a synchrotron, or a storage ring, the closed orbit of a stored charged particle depends on its energy deviation from the design value. The existence of this dispersion inevitably yields dynamic coupling between the horizontal coordinate and longitudinal momentum of the particle, thus making the beam behavior more complicated. If the dispersive effect is negligible, then ion traps and storage rings become almost equivalent [14], which means that we encounter no substantial obstacle toward Coulomb crystallization. In general, however, strong momentum dispersion is inevitable as far as regular storage rings are concerned. This is one primary reason why the crystallization of fast stored beams is so difficult and has not been accomplished yet.

The cooling force provided by a usual cooling device is designed so as to equalize the longitudinal velocities of all stored ions, but such a force is not suitable for a 3D crystalline state with finite horizontal extent because of the shearing force, as illustrated in Fig. 1. In order to maintain a crystalline structure, stored particles must have an identical "angular" velocity rather than an identical "linear" velocity; in other words, we must compensate the difference in the revolution frequencies. As a solution 


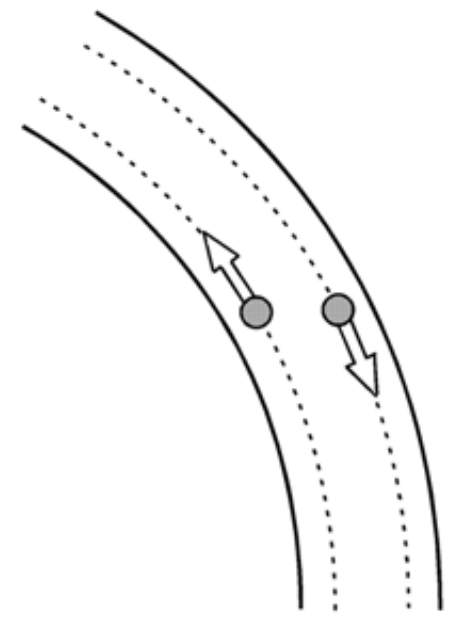

FIG. 1. The conceptual illustration of the shearing force. If all stored particles in a crystalline ground state have the same longitudinal velocity, the revolution frequency of a radially outer particle becomes longer than that of an inner particle. Consequently, the two particles are more and more distanced longitudinally every turn, which eventually leads to the melting of the crystalline state. The strength of the shearing force is closely related to the momentum dispersion, as shown in Sec. III.

of such a problem, tapered cooling has been proposed [12]. In tapered cooling, particles at different radial positions are cooled towards different velocities in order to realize the same angular momentum. However, no practical method to generate a tapered light has been known. In this paper, we propose an alternative scheme to stabilize 3D crystals, namely, a storage ring that has deflection elements using magnetic field and electric field simultaneously.

As first pointed out in Refs. [15,16], it is possible to suppress the momentum dispersion by adding a horizontal electrostatic field $\mathbf{E}$ in addition to the bending magnetic field $\mathbf{B}$. Such a deflection element is realized by combining a dipole magnet and a cylindrical electrostatic deflector. The electric force must be directed outward (Fig. 2), namely, opposite to the direction of the magnetic force. In order to eliminate the linear dispersion, we require the following relation to be satisfied:

$$
\left(1+\frac{1}{\gamma_{0}^{2}}\right) \mathbf{E}\left(\rho_{0}\right)=-\mathbf{v}_{0} \times \mathbf{B}
$$

where $\gamma_{0}$ is the Lorentz factor, $\mathbf{v}_{0}$ is the velocity of the reference particle, and $\rho_{0}$ is the radius of curvature of the design orbit in the bending region. The radial electric field $\mathbf{E}$ can be provided by a cylindrical electrostatic deflector as depicted in Fig. 2. Ideally, the bending electric field is given by

$$
\mathbf{E}\left(\rho_{0}\right)=\frac{V_{0}}{\rho_{0}+x},
$$

where $V_{0}$ is a constant and $x$ is the horizontal coordinate measured from the design orbit. Employing such dispersion suppressors, we can construct a unique storage ring in

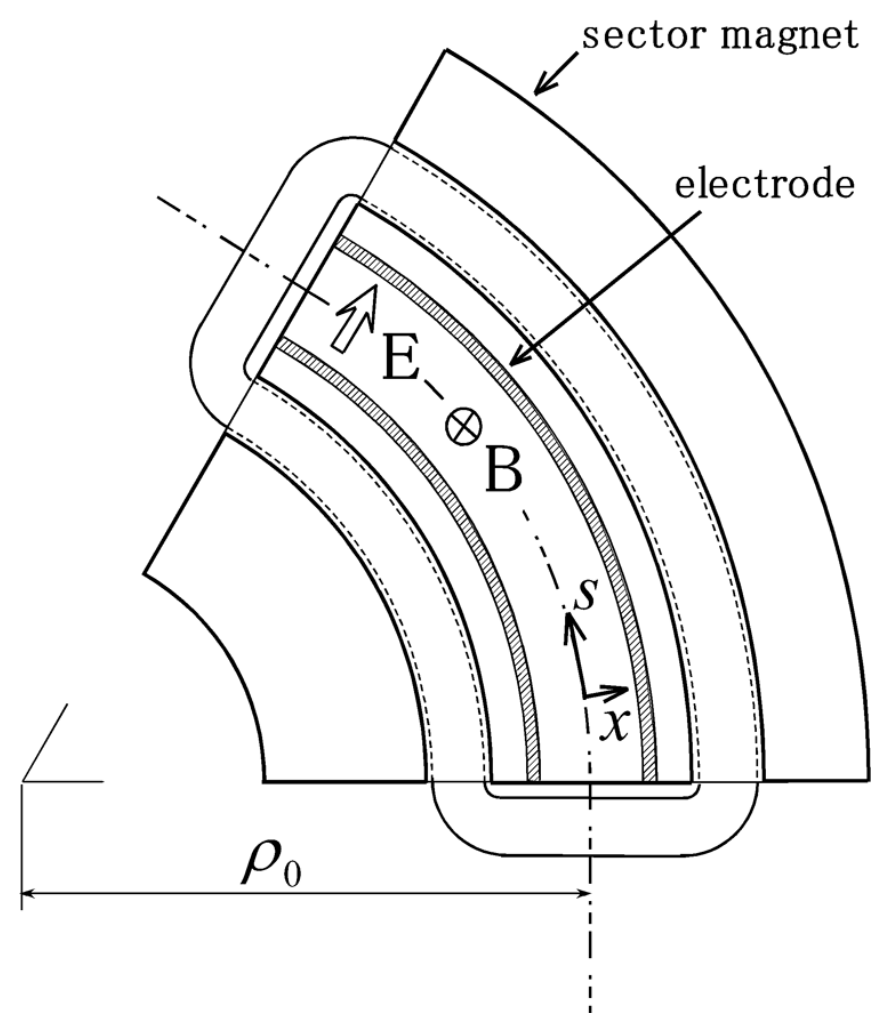

FIG. 2. Example of a dispersion suppressor. A curved electrostatic deflector is installed in the gap of a dipole magnet. Note that the direction of the electric field is radially outward.

which the momentum dispersion is canceled throughout the whole circumference by adjusting the ratio of the electric and magnetic field strengths.

As we prove later, the scalar potential of the dispersion suppressor causes the longitudinal acceleration (or deceleration) of particles, automatically equalizing their angular velocities in the bending region. This means that, in a storage ring constructed by such deflectors, it is likely that 3D crystalline beams can be produced.

A small laser-equipped storage ring (S-LSR) is now under construction at the ICR Kyoto University in collaboration with the National Institute of Radiological Sciences [17]. Laser and electron cooling techniques are to be applied, at S-LSR, to ion beams that have relatively low kinetic energies. The dispersion suppressor described above is also used in order to demonstrate the practicability and potential of a dispersionless system. As a possible application of the dispersion-free operating mode, the laser cooling of a ${ }^{24} \mathrm{Mg}^{+}$beam is planned.

This paper is organized as follows: In Sec. II, we consider the single-particle dynamics in the dispersion suppressor. The Hamiltonian of a storage ring constructed by dispersion suppressors is derived in Sec. III. In this section, it is shown that the heating mechanism due to the shearing force disappears at the dispersion-free condition. It is also shown that the Hamiltonian of the dispersion-free storage ring becomes equivalent to that of a linear ion trap. We then 
show, in Sec. IV, the actual design of the deflector installed in S-LSR. The lattice functions in different operating modes and the stability region of betatron motion are also exhibited in this section. Considerations related to the formation of a 3D crystal, both in general and then in detail for the S-LSR ring, are presented in Sec. IV, and summarized in Sec. V.

\section{DISPERSION SUPPRESSOR}

The Hamiltonian of the dispersion suppressor is derived in the Frenet-Serret coordinate system [18]. Choosing the path length $s$ as the independent variable, we obtain the relativistic Hamiltonian that governs the motion of a charged particle in a bending region where not only a dipole magnetic field but also an electric field for dispersion compensation is present, of the form [19]

$$
\begin{aligned}
H= & -\left(1+\frac{x}{\rho_{0}}\right) \sqrt{\left(\frac{p_{t}+q \phi_{D}}{c}\right)^{2}-m^{2} c^{2}-p_{x}^{2}-p_{y}^{2}} \\
& -q\left(1+\frac{x}{\rho_{0}}\right) A_{s},
\end{aligned}
$$

where $m$ and $q$ are the rest mass and charge state of particles, $c$ is the speed of light, $\phi_{D}$ is the scalar potential in the electrostatic deflector, $p_{t}$ is the longitudinal canonical momentum conjugate to time $t$, and we have assumed that the vector potential $\mathbf{A}$ only has the longitudinal component, i.e., $\mathbf{A}=\left(0,0, A_{s}\right)$. By expanding the square root and leaving only low-order terms, Eq. (3) becomes

$$
H=-\left(1+\frac{x}{\rho_{0}}\right) q A_{s}-\left(1+\frac{x}{\rho_{0}}\right) p+\frac{p_{x}^{2}+p_{y}^{2}}{2 p},
$$

where $p=m \beta \gamma c=\sqrt{\left(p_{t}+q \phi\right)^{2} / c^{2}-m^{2} c^{2}}$ with $\beta$ being the normalized velocity by the light speed $c$. The scalar potential in the electrostatic deflector which is set along the design orbit can be expressed as

$$
\phi_{D}=-V_{0} \ln \left(1+\frac{x}{\rho}\right)=V_{0} \sum_{n=1}^{\infty} \frac{(-1)^{n}}{n}\left(\frac{x}{\rho_{0}}\right)^{n} .
$$

The vector potential of the bending magnet is given by

$$
A_{s}=-\frac{B_{y}}{2}\left(\rho_{0}+x\right) \text {, }
$$

where $B_{y}$ is the dipole magnetic field. Note that the equilibrium kinetic momentum $p_{0}\left(\equiv m \beta_{0} \gamma_{0} c\right)$ is not equal to $q B_{y} \rho$ due to the existence of the bending electric field. Since the electric field strength is $V_{0} / \rho_{0}$ along the design orbit, the equilibrium kinetic momentum becomes

$$
p_{0}=q B_{y} \rho_{0}-\frac{q V_{0}}{\beta_{0} c} .
$$

The momentum deviation from the design momentum $p_{0}=m \beta_{0} \gamma_{0} c=\sqrt{E_{0}^{2} / c^{2}-m^{2} c^{2}}$ can approximately be written as

$$
\Delta p \equiv p-p_{0} \approx \frac{\Delta E-q \phi_{D}}{\beta_{0} c}-\frac{1}{2 p_{0}}\left(\frac{\Delta E-q \phi_{D}}{\beta_{0} c \gamma_{0}}\right)^{2},
$$

where $\Delta E$ is the energy deviation from the design value $-\Delta E=m \gamma_{0} c^{2}-\left(-p_{t}\right)$. Inserting Eq. (6) and $p=p_{0}+$ $\Delta p$ into Eq. (4) and neglecting nonlinear terms, one finds

$$
\begin{aligned}
\tilde{H}= & \frac{q V_{0}}{\beta_{0}^{2} E_{0}} \frac{x}{\rho_{0}}+\frac{1}{2}\left(1+\frac{q V_{0}}{\beta_{0}^{2} E_{0}}\right)\left(\frac{x}{\rho_{0}}\right)^{2}-\left(1+\frac{x}{\rho_{0}}\right) \frac{\Delta p}{p_{0}} \\
& +\frac{\tilde{p}_{x}^{2}+\tilde{p}_{y}^{2}}{2},
\end{aligned}
$$

where the transverse momenta have been scaled to be dimensionless; namely, $\tilde{p}_{x(y)}=p_{x(y)} / p_{0}$. Substitution of Eq. (8) together with Eq. (5) into Eq. (9) yields the approximate Hamiltonian

$$
\begin{aligned}
\tilde{H}= & -\frac{\Delta E}{\beta_{0}^{2} E_{0}} \frac{x}{\rho_{0}}\left(1-\frac{q V_{0}}{\gamma_{0}^{2} \beta_{0}^{2} E_{0}}\right)-\frac{\Delta E}{\beta_{0}^{2} E_{0}}+\frac{1}{2 \gamma_{0}^{2}}\left(\frac{\Delta E}{\beta_{0}^{2} E_{0}}\right)^{2} \\
& +\frac{\tilde{p}_{x}^{2}+\tilde{p}_{y}^{2}}{2}+\left[1+\frac{1}{\gamma_{0}^{2}}\left(\frac{q V_{0}}{\beta_{0}^{2} E_{0}}\right)^{2}\right] \frac{x^{2}}{2 \rho_{0}^{2}} .
\end{aligned}
$$

From this Hamiltonian, we obtain the horizontal equation of motion in the bending region

$$
\frac{d^{2} x}{d s^{2}} \approx-\left[1+\frac{1}{\gamma_{0}^{2}}\left(\frac{q V_{0}}{\beta_{0}^{2} E_{0}}\right)^{2}\right] \frac{x}{\rho_{0}^{2}}+\frac{1}{\rho_{0}}\left(1-\frac{q V_{0}}{\gamma_{0}^{2} \beta_{0}^{2} E_{0}}\right) \frac{\Delta E}{\beta_{0}^{2} E_{0}} .
$$

Clearly, the last term in the right-hand side of Eq. (11) gives rise to linear dispersion, and its coefficient $1 / \rho_{0}(1-$ $\left.q V_{0} / \gamma_{0}^{2} \beta_{0}^{2} E_{0}\right)$ decides the strength of the dispersion. It is thus possible to control momentum dispersion over a wide range by changing the electric and magnetic field strengths with the condition (7) fulfilled. In particular, dispersive effects can be minimized provided that

$$
\frac{q V_{0}}{\gamma_{0}^{2} \beta_{0}^{2} E_{0}}=1
$$

which leads, by using Eq. (7), to $V_{0} / \rho=B_{y} \cdot[\beta c /(2-$ $\left.\left.\beta^{2}\right)\right]$. This equation indicates that, when the beam energy is high, a very large voltage is needed to compensate dispersive effects. Therefore, the present dispersion suppressor is relevant only to low-energy beams. From Eqs. (7) and (12), we find $\left(1+1 / \gamma_{0}^{2}\right) V_{0} / \rho_{0}=B_{y} \beta_{0} c$ that is identical to the condition (1). When the dispersion-free condition (12) is satisfied, the horizontal equation of motion in the bending region becomes

$$
\frac{d^{2} x}{d s^{2}} \approx-\frac{1+\gamma_{0}^{2}}{\rho_{0}^{2}} \cdot x
$$

where the horizontal motion has been decoupled from the longitudinal motion in linear approximation. It is possible to construct a dispersion-free storage ring by using the dispersion suppressors. 


\section{APPLICATION TO A STORAGE RING}

For the transport of charged particles, dispersion-free deflection elements were proposed in $[15,16]$. But this scheme has never been used in the design of synchrotrons and storage rings up to now, because the required electric fields become too large for particle beams used in these accelerators. We propose the possibility of incorporating this scheme in a low-energy storage ring. In this section, we show some characteristics of a storage ring constructed with dispersion suppressors.

\section{A. Hamiltonian of a storage ring}

A beam circulating in a dispersion-free storage ring receives only the alternating focusing force, thus, one can expect that the beam dynamics of the dispersion-free ring coincides with that of a linear beam transport line or a linear ion trap. In this section, the Hamiltonian of the storage ring constructed with dispersion suppressors is investigated. Here, a separated function type lattice is assumed. When quadrupole magnets and an rf cavity are taken into account in addition to dispersion suppressors, the vector potential (6) becomes

$A_{s}=-\frac{B_{y}}{2}(\rho+x)+\frac{1}{2} B_{1}\left(x^{2}-y^{2}\right)+\delta_{p}(s) \frac{V_{\mathrm{rf}}}{\omega} \cos \left(\omega t+\phi_{0}\right)$,

where $B_{1}$ is the gradient of the quadrupole field. $V_{\mathrm{rf}}$ and $\phi_{0}$ are a voltage amplitude and an initial phase of the rf cavity, respectively, and $\delta_{p}(s)$ is a periodic delta function. Since a storage ring is considered now, the rf frequency $\omega$ is constant. The rf cavity is installed in the straight section at the coordinate $s=0$.

When Eq. (14) and $p=p_{0}+\Delta p$ are inserted into Eq. (4) and nonlinear terms are neglected, one finds

$$
\begin{aligned}
\tilde{H}= & -\frac{\Delta E}{\beta_{0}^{2} E_{0}} \frac{x}{\rho}\left(1-\frac{q \kappa}{\gamma_{0}^{2} \beta_{0}^{2} E_{0}}\right)-\frac{\Delta E}{\beta_{0}^{2} E_{0}}+\frac{1}{2 \gamma_{0}^{2}}\left(\frac{\Delta E}{\beta_{0}^{2} E_{0}}\right)^{2} \\
& +\frac{\tilde{p}_{x}^{2}+\tilde{p}_{y}^{2}}{2}+\frac{1}{2}\left(K_{x} x^{2}+K_{y} y^{2}\right)-\delta_{p}(s) \frac{q V_{\mathrm{rf}}}{p_{0} \omega} \cos \left(\omega t+\phi_{0}\right),
\end{aligned}
$$

where

$$
K_{x}=\frac{1}{\rho_{0}^{2}}\left[1+\frac{1}{\gamma_{0}^{2}}\left(\frac{q \kappa}{\beta_{0}^{2} E_{0}}\right)^{2}\right]-\frac{q K_{1}}{p_{0}}, \quad K_{y}=\frac{q K_{1}}{p_{0}} .
$$

$\rho, \kappa$, and $K_{1}$ are functions of $s ; \rho=\rho_{0}, \kappa=V_{0}$ in the bending region, and $\rho=\infty, \kappa=0$ in other regions. $K_{1}$ is $B_{1}$ in the quadrupole magnet region, and $K_{1}=0$ in other regions.

\section{B. Effect of the electrostatic potential}

The electrostatic potential of the dispersion suppressor causes the energy transfer between kinetic energy and potential energy of charged particles while conserving the total energy. Here, the total energy means summation of the kinetic energy and potential energy and it corresponds to $E$ in the notation used in this paper. One can find that $\Delta E$ is also an approximate constant of motion, if the $\mathrm{rf}$ voltage is not imposed [see Eq. (15)]. On the other hand, the kinetic energy and the momentum may not be constant, in contrast with a conventional magnetic storage ring.

Suppose an ion beam is strongly cooled by a cooling force in a storage ring. If the design orbit is linear, the momentum spread eventually vanishes, i.e., $\Delta p=0$, at low temperature limit where the beam is Coulomb crystallized. As shown in Refs. [10,20], when the density of the beam is low, the crystalline structure is a $1 \mathrm{D}$ chain. If the density is larger than the 1D state, a 2D crystalline structure is developed in the weaker focusing direction. If the density is enhanced more, the particles naturally arrange into a $3 \mathrm{D}$ crystal structure, and the beam has a finite horizontal extent. In an ordinary storage ring, however, the condition $\Delta p=0$ does not meet the stability requirement of a crystalline state with a finite horizontal extent because the bending magnets generate momentum dispersion; as briefly discussed in Sec. I, a radially outer particle must travel slightly faster than the inner particles, so as to realize a condition such that the angular velocities of all particles, rather than the linear velocity, are identical. The stability of a multidimensional crystalline beam is, therefore, not guaranteed unless the cooling force is "tapered" [12]. By contrast, the present dispersion-free system can compensate the difference of the angular velocities even if the cooling force is not tapered, because of the above energy transfer mechanism. However, in order to realize this condition, it is essential that the ring has a straight section, in addition to satisfying a dispersion-free condition. The reason is as follows.

First, for pedagogical purposes, we consider a dispersion-free ring with a constant bending field, i.e., the whole circumference of the ring is occupied by the bending element. The cooling force acts so that the momentum spread approaches zero, and the momentum is almost constant in this ring, like that of conventional rings. If the beam has finite horizontal extent, this situation is not suitable for the condition of a 3D crystal beam; namely, all particles do not have the same angular momentum. This ring is merely dispersion free.

Next, we consider a dispersion-free ring which has straight sections. In such a ring, the momentum is not constant because of the acceleration (or deceleration) of the charged particle at the entrance (or exit) of the bending section. The relation between the deviation of the total energy $\Delta E$ and the momentum deviation $\Delta p$ is given by Eq. (8). If a beam is cooled at a straight section, the momentum deviation $\Delta p$ becomes zero and the energy deviation $\Delta E$ also becomes zero because of the zero scalar potential. When a beam with a finite horizontal extent enters into the bending region, the particles receive some kinetic energy gain (loss) from the deflection electric field 
in exchange for loss (gain) of the potential energy. The amount of the gain depends on the horizontal coordinate $x$. When $\left|x / \rho_{0}\right| \ll 1$, Eq. (5) gives $\phi_{D} \approx-V_{0} \cdot x / \rho_{0}$ and, accordingly, the potential along an inner orbit is higher than that along an outer orbit; in other words, a particle traveling in the region of negative $x$ (positive $x$ ) is decelerated (accelerated) at the entrance of the electrostatic deflector. At the exit, the opposite effect takes place, and the particle recovers the original kinetic energy before entering the bending region. Now, $\Delta E$ is zero, because of the cooling at the straight section, and it is zero all around the ring, since it is constant of motion. Then, if once an ideal ground state [21] is reached, from Eq. (8), one obtains the relation $p \approx p_{0}+\left(q V_{0} / \beta_{0} c \rho_{0}\right) \cdot x$. This relation can be rewritten, with the condition (12), as $p / p_{0} \approx$ $1+\gamma^{2} x / \rho_{0}$ or, equivalently,

$$
\frac{\nu}{\nu_{0}} \approx 1+\frac{x}{\rho_{0}},
$$

where $\nu$ denotes the velocity of a particle at the horizontal position $x$, and $\nu_{0}=\beta_{0} c$. It is now evident that the angular velocities of all particles are approximately the same. We can thus suggest that the stability of a three-dimensional crystalline beam is greatly improved in the dispersion-free storage ring presented in this paper.

Even if the storage ring satisfies the dispersion-free condition, making particles to have the same angular velocity is impossible without the straight section. Thus, it is essential to "cool at the straight section (which has no electrostatic potential)" and to cause the acceleration (or deceleration) of the charged particle at the entrance (or exit) of the bending section.

\section{Analytical treatment of the shearing force}

As shown in the previous subsection, the effect of acceleration (or deceleration) at the boundary of the bending section plays an important role. This effect is included in the Hamiltonian formalism automatically in this paper. Therefore, we can show these effects analytically. The first term of the Hamiltonian (15) yields dynamic coupling between the horizontal coordinates and longitudinal momenta. From the derivation process of the horizontal equation of motion (11), one finds this shear term [22] generates the dispersion term of the equation of motion. We can show the shear term also generates the shearing force. In order to show the effect of the shear term explicitly, the rf cavity is switched off. When the relative time $\Delta t=t-s / \beta_{0} c$ is introduced, from the Hamiltonian (15), longitudinal equations of motion are given by

$$
\begin{gathered}
\frac{d(\Delta t)}{d s}=\frac{1}{\beta_{0} c}\left[\frac{x}{\rho}\left(1-\frac{q \kappa}{\gamma_{0}^{2} \beta_{0}^{2} E_{0}}\right)-\frac{1}{\gamma_{0}^{2}}\left(\frac{\Delta E}{\beta_{0}^{2} E_{0}}\right)\right], \\
\frac{d}{d s}\left(\frac{-\Delta E}{p_{0}}\right)=0 .
\end{gathered}
$$

The first term on the right-hand side of Eq. (17) represents the shearing force, and it is generated from the shear term. The second term represents the difference of the revolution time caused by the energy deviation. According to Eq. (17), in a crystalline state (where $\Delta E=$ 0 , it is constant in the ring) with a finite horizontal extent $x$, the difference of the revolution time is dominated by the shearing force and its strength is proportional to the strength of the dispersion; $1 / \rho\left(1-q \kappa / \gamma_{0}^{2} \beta_{0}^{2} E_{0}\right)$.

For the dispersion-free condition (12), the shearing force canceled out and the revolution time does not depend on the horizontal extent of the beam $x$. Therefore, in a storage ring constructed with dispersion suppressors, the cancellation of the shearing force is synonymous with the cancellation of the dispersion.

The shear term is caused by the geometric factor of bending; namely, this term exists because of the finite bending radius $\rho$. This term causes a crucial difference between the Hamiltonians of the storage ring and the linear ion trap. Fortunately, this term is canceled out at the dispersion-free condition. Then, although there are bending sections, the dynamics of the particle becomes the same as the straight section. Therefore, in the dispersionfree condition, the Hamiltonian (15) becomes equivalent to the Hamiltonian of an rf linear ion trap, on the beam rest frame. Thus, the beam behavior in the dispersion-free ring, except for higher-order nonlinear terms, is similar to that in the linear ion trap in which a multidimensional crystal structure has been observed experimentally [23]. The discussion about nonlinear terms is performed in Sec. IV.

\section{Effect of the rf cavity}

When the rf voltage is imposed, only the longitudinal equation of motion (18) is corrected [24]

$$
\frac{d(\Delta E)}{d s}=\frac{q V_{\mathrm{rf}}}{C} \sin \phi
$$

where the synchrotron phase $\phi=\Delta \phi+\phi_{0}=\omega \Delta t+\phi_{0}$ is introduced and $C$ is the length of reference orbit in the ring. In the following, the initial phase of the rf cavity $\phi_{0}$ is set to zero so that the reference particle may not have energy gain and synchrotron oscillation. Then, Eq. (19) gives an energy change so that the particles which are deviated from the design phase experience oscillation.

In a low beam current limit in which the space charge effect is negligible, the longitudinal equation of motion of the dispersion-free storage ring becomes the synchrotron equation which has phase slip factor $\eta=-1 / \gamma_{0}^{2}$. Thus the synchrotron oscillation is stable. This result is reasonable, because the phase slip factor is defined by $\eta=\alpha-1 / \gamma_{0}^{2}$ and the momentum compaction factor of the dispersionfree ring is zero; $\alpha=1 / C \oint D_{x}(s) / \rho(s) d s=0$. Here $D_{x}(s)$ is the horizontal dispersion function of the ring, and it is zero all around the dispersion-free ring. This result 
means the dispersion-free ring has infinite high transition energy; $\gamma_{t}=1 / \sqrt{\alpha}$.

As shown in Ref. [11], there are so-called maintenance conditions for realization of a crystal beam in a storage ring. The first maintenance condition is that the beam energy must be below the transition energy; $\gamma<\gamma_{t}$. The second condition is $N>2 \sqrt{2} \nu_{T}$, where $N$ is the number of the superperiod of the ring, and $\nu_{T}$ is the transverse tune. The dispersion-free ring satisfies the first condition in principle. If the dispersion-free storage ring has a larger number of superperiods or a large bending radius, it is thought to satisfy the second condition in general. Consideration of the second maintenance condition is described in Sec. IV B.

In the following, we consider the crystalline state, in order to explain analytically the heating mechanism of the crystalline beam induced by the dispersion. In a 3D crystalline state, betatron oscillation is strongly suppressed, and the particles no longer oscillate across the reference orbit. This means such particles always have the same sign of $x$ in Eq. (17). If a bunched 3D crystalline beam continues to be cooled by a cooling force in the straight section, the energy spread $\Delta E$ of Eq. (17) approaches zero. But, in the bending region, the deviations of the synchrotron phases $\Delta \phi=\omega \Delta t$ of radially outer and inner particles are increased because of the first term of Eq. (17) (shearing force), if the dispersion-free condition is not satisfied. This increase of the synchrotron phase deviation $\Delta \phi$ affects the energy spread $\Delta E$ through Eq. (19). Eventually, the energy spread is extended by the rf potential through Eq. (19). Finally, this heating rate balances with the cooling rate of the cooling force. Because of such a mechanism, the reachable temperature of the $3 \mathrm{D}$ crystalline beam is limited.

In the dispersion-free condition, this heating mechanism is completely suppressed due to the cancellation of the first term of Eq. (17), and the stability of the 3D crystalline beam is greatly improved. In this state, the energy spread $\Delta E$ of the final equilibrium state becomes zero. As shown in Ref. [13], the synchrotron oscillation of the crystalline beam is strongly suppressed despite the finite bunch length (finite phase deviation $\Delta \phi$ ). This is the same reason as the suppression of the betatron oscillation of a 3D crystalline beam which has a finite transverse extent. Thus, the synchrotron motion of the $3 \mathrm{D}$ crystalline beam is suppressed completely.

In order to realize a strong three-dimensional laser cooling, the method of utilizing a synchrobetatron coupling induced by the rf cavity [25] has been proposed. However, in the dispersion-free storage ring, it is difficult to generate synchrobetatron coupling by a normal rf cavity, because there is no dispersion. In the dispersion-free case, we can use a coupling rf cavity $[13,26]$ for the $3 \mathrm{D}$ cooling. The coupling rf cavity scheme realizes the synchrobetatron coupling by using a special mode of the rf electric field which depends on the transverse position. For a low-energy storage ring, even in the dispersion-free case, the coupling rf cavity can create the enough coupling strength for 3D cooling. The energy transfer between longitudinal motion and transverse motion becomes comparable, in order, to that of the energy of the laser photon for cooling, by reasonable applied voltage to the coupling cavity.

\section{ION STORAGE RING S-LSR}

A small laser-equipped storage ring has been developed by the collaborations ICR Kyoto University and National Institute of Radiological Sciences. In S-LSR, electron cooling and laser cooling will be used to increase the phase space density of the stored ion beam. One of the purposes is to demonstrate the feasibility of crystallizing a stored heavy ion beam [17]. The main parameters are listed in Table I. The lattice design is shown in Fig. 3. In this section, a concrete method to realize a dispersion-free ring is described taking the case of S-LSR as an example, and the effects of the dispersion suppressor in the lattice of S-LSR are shown.

\section{A. Elements of S-LSR}

The deflection elements for S-LSR have a structure as shown in Fig. 4. Electrostatic deflectors are installed in all the dipole magnets for the purpose of dispersion compensation. The electric and magnetic fields needed for dispersion-free storage of a $35 \mathrm{keV}^{24} \mathrm{Mg}^{+}$beam are $6.7 \times$ $10^{4} \mathrm{~V} / \mathrm{m}$ and $0.252 \mathrm{~T}$, respectively. The electrostatic deflectors are designed to be moved away radially, so that the ring can be operated with the normal dispersion as well (Fig. 4). Since the electrostatic deflector is to be installed in the small gap of the dipole magnet, the vertical aperture is limited to $26 \mathrm{~mm}$ (Fig. 5). Furthermore, the electric field of the electrostatic deflector is reduced by the influence of the wall of the vacuum vessel. By introducing intermediate electrodes, the strength of the electric field at the center of the aperture is maintained [27]. The horizontal aperture has been determined as $30 \mathrm{~mm}$ compromising the available aperture for beam injection or circulation and the deviation of the radial distribution of the electric field. Namely, as the horizontal aperture becomes wider, it becomes better for the beam injection and circulation, but the deviation of the radial distribution of the field from the ideal distribution [Eq. (2)] becomes larger.

The momentum spread of the injected ${ }^{24} \mathrm{Mg}^{+}$beam with the kinetic energy of $35 \mathrm{keV}$ is expected to be less than $10^{-3}$. The emittance of the ${ }^{24} \mathrm{Mg}^{+}$beam which is directly pulled out from the ion source is estimated to be about $40 \pi \mathrm{mm}$ mrad. The ${ }^{24} \mathrm{Mg}^{+}$beam will be injected into the ring, without further acceleration. Since the aperture of the electrostatic deflector is small, the small part of emittance of the beam is selected by a double slit. The emittance of the injected beam is to be adjusted to the size of 1 to $10 \pi \mathrm{mm} \mathrm{mrad}$ by the double slit. Dipole magnets for S- 
TABLE I. Main parameters of S-LSR (storage of ${ }^{24} \mathrm{Mg}^{+}$)

\begin{tabular}{lc}
\hline \hline \multicolumn{1}{c}{ Quantity } & Value \\
\hline Ions to be laser cooled & ${ }^{24} \mathrm{Mg}^{+}$ \\
Total kinetic energy & $35 \mathrm{keV}$ \\
Circumference & $22.557 \mathrm{~m}$ \\
Radius of curvature at the bending section & $1.05 \mathrm{~m}$ \\
Number of deflection elements & 6 \\
Deflection angle of each deflection element & $60^{\circ}$ \\
Gap height of the dipole magnet & $70 \mathrm{~mm}$ \\
\hline \hline
\end{tabular}

LSR have already been manufactured and their evaluation by the field measurement has also been completed. The design of electrostatic deflectors for S-LSR is finished and they are now being manufactured.

The rf cavity shown in Fig. 3 is to be used for beam bunching. When S-LSR is used as a magnetic storage ring, this rf cavity also is to be used for the 3D cooling; its parameters at the operating point $(1.44,1.44)$ are shown in Table II. At this operating point, the needed values of the rf voltage and the harmonic number become very large. Therefore the 3D cooling will be performed at the operating point $(2.067,1.073)$, rather than this operating point. For the dispersion-free mode of S-LSR, the rf cavity can be used only for beam bunching. The phase slip factor of the dispersion-free mode is always $\eta=-1$; on the other hand,

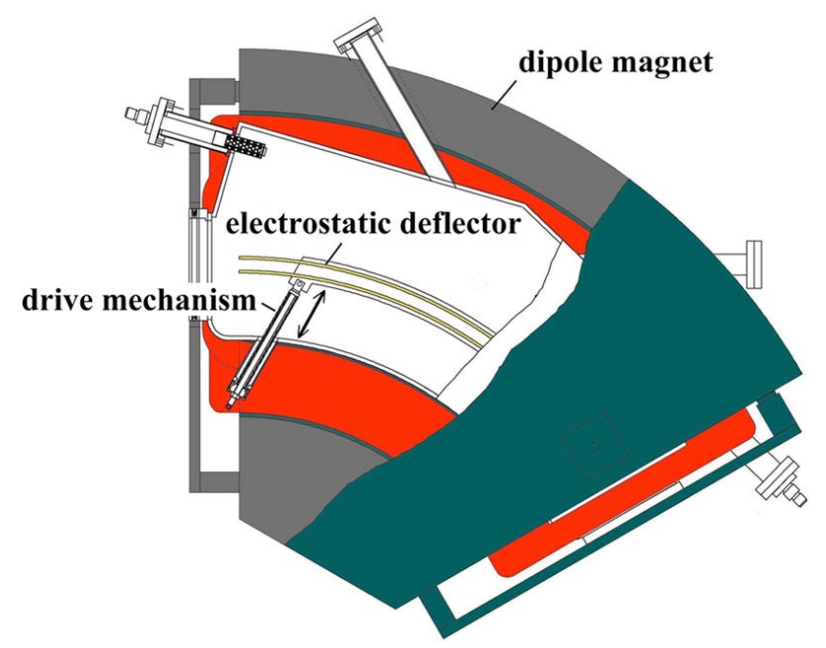

FIG. 4. (Color) Deflection element for S-LSR. The deflection element is constructed by a dipole magnet which has no field gradient and a curved electrostatic deflector. The electrostatic deflector is installed in the gap of the dipole magnet. The electrostatic deflector can be moved out when the deflection element is used as a normal dipole magnet.

the absolute value of $\eta$ of the magnetic mode is usually less than 1 . Therefore, at the dispersion-free condition, the rf voltage required for the same synchrotron tune is lower than that of the magnetic mode. In order to realize the $3 \mathrm{D}$

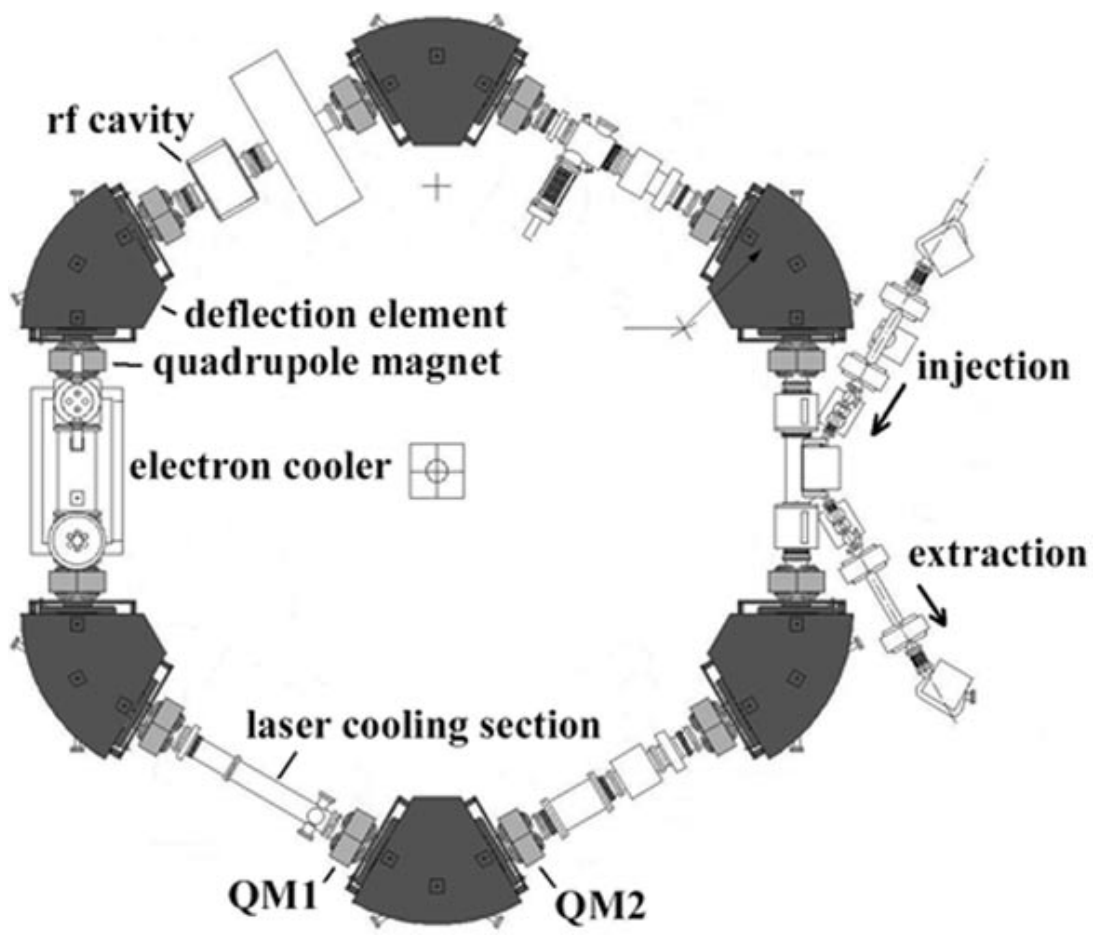

FIG. 3. The lattice structure of S-LSR. The number of the superperiod is 6. An rf cavity is introduced for beam bunching. Especially, in the case of only a magnetic storage ring, the rf cavity is also used for 3D cooling. For the dispersion-free condition, in addition to the rf cavity, a coupling rf cavity is to be installed for 3D cooling. In the horizontal beam dynamics, the radial focusing effect of the deflection element is utilized for beam focusing. Thus, all quadrupole magnets have diverging effect horizontally and focusing effect vertically. 
cooling in the dispersion-free mode, in addition to the rf cavity for beam bunching, a coupling rf cavity will be added. The design of the coupling rf cavity needs further investigation.

\section{B. Transfer matrix formula}

In this subsection, the lattice parameters of S-LSR are calculated by the transfer matrix analysis [28]. The

$$
M=\left(\begin{array}{cc}
\cos \sqrt{k} L & \frac{1}{\sqrt{k}} \sin \sqrt{k} L \\
-\sqrt{k} \sin \sqrt{k} L & \cos \sqrt{k} L \\
0 & 0 \\
0 & 0 \\
-\frac{1}{\beta_{0}} \frac{d}{\sqrt{k}} \sin \sqrt{k} L & -\frac{1}{\beta_{0}} \frac{d}{k}(1-\cos \sqrt{k} L) \\
0 & 0
\end{array}\right.
$$

In this transfer matrix, $L$ denotes the path length of the reference particle in the dispersion suppressor, and the following notations are introduced to simplify the formula:

$$
k=\left[1+\frac{1}{\gamma_{0}^{2}}\left(\frac{q V_{0}}{\beta_{0}^{2} E_{0}}\right)^{2}\right] \frac{1}{\rho_{0}^{2}}, \quad d=\left(1-\frac{q V_{0}}{\gamma_{0}^{2} \beta_{0}^{2} E_{0}}\right) \frac{1}{\rho_{0}} .
$$

First, the stable region of the betatron oscillation is calculated. For the dispersion-free condition, the stable

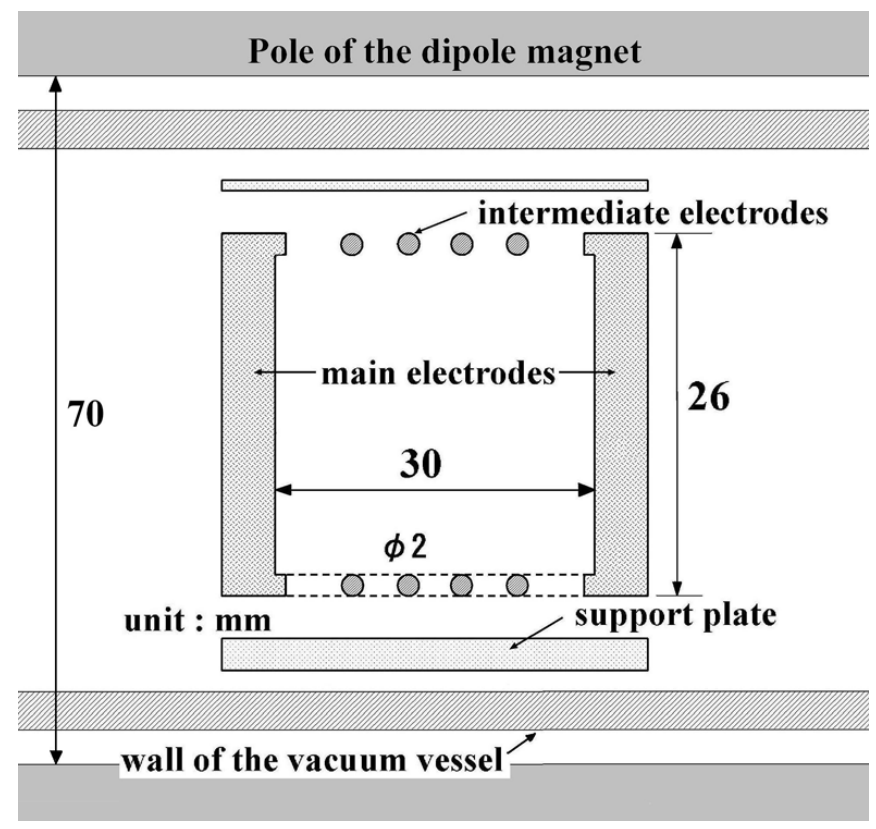

FIG. 5. Cross section around the electrostatic deflector. The height of the electrostatic deflector is limited by the gap size of the dipole magnet and the vacuum vessel. The electrostatic deflector is constructed by a pair of main electrodes and four pairs of intermediate electrodes. The ideal field distribution is maintained by the intermediate electrodes. In addition to the electrodes, support plates and ceramic plates are introduced for the purpose of keeping the position of the electrodes. transfer matrix acts on the phase space coordinates $\left(x, \tilde{p}_{x}, y, \tilde{p}_{y},-c \Delta t, W\right)$, where $W=\Delta E / \beta_{0} E_{0}$. The transfer matrix of the dispersion suppressor is derived by solving the canonical equation from the Hamiltonian (10). From the canonical equation, one easily derives the relations between the slopes $x^{\prime}, y^{\prime}$ and the normalized canonical momenta $\tilde{p}_{x}, \tilde{p}_{y} ; x^{\prime}=\tilde{p}_{x}, y^{\prime}=\tilde{p}_{y}$. Then the transfer matrix becomes

$\left.\begin{array}{cccc}0 & 0 & 0 & \frac{1}{\beta_{0}} \frac{d}{k}(1-\cos \sqrt{k} L) \\ 0 & 0 & 0 & \frac{1}{\beta_{0}} \frac{d}{\sqrt{k}} \sin \sqrt{k} L \\ 1 & L & 0 & 0 \\ 0 & 1 & 0 & 0 \\ 0 & 0 & 1 & -\frac{1}{\beta_{0}^{2}} \frac{d^{2}}{k}\left(L-\frac{1}{\sqrt{k}} \sin \sqrt{k} L\right)+\frac{L}{\beta_{0}^{2} \gamma_{0}^{2}} \\ 0 & 0 & 0 & 1\end{array}\right)$.

region is shown in Fig. 6. The field gradient of QM1 and QM2 in Fig. $3\left(k_{1}\right.$ and $\left.k_{2}\right)$ are used as the parameters. The field gradients $k_{1}$ and $k_{2}$ are normalized by the magnetic rigidity of the $35 \mathrm{keV}^{24} \mathrm{Mg}^{+}$beam; $k=B_{1} / B_{0} \rho_{0}$. The stable regions of betatron oscillation are drawn by selecting regions which satisfy the condition that the absolute value of the trace of the transverse transfer matrix of one superperiod is less than 2 . At the operating point $A 1$ of Fig. 6 , the betatron tunes become $(1.49,3.49)$, and the value of the beta functions becomes minimum in the stable region. The beta functions of this operating point are shown in Fig. 7 as the functions of the position $s$ along the reference orbit. The lattice parameters of the operating point $A 1$ are compared to those of magnetic mode. In the case of only magnetic field, the horizontal and vertical tunes are set to $(1.44,1.44)$. In this case, beta functions become as shown in Fig. 8.

We have found that at almost all the usable operation points, the dispersion-free case has a larger $\beta_{x}$ than in the magnetic case and the vertical tune is enhanced. The reason for this can be found from Eqs. (7) and (11). One finds that the radial focusing of the dispersion suppressor can be controllable without changing the bending radius by changing the ratio of the two bending fields, and, at the

TABLE II. Main parameters of the rf cavity (3D cooling at the magnetic mode).

\begin{tabular}{lc}
\hline \hline \multicolumn{1}{c}{ Quantity } & Value \\
\hline Ions to be laser cooled & ${ }^{24} \mathrm{Mg}^{+}$ \\
Total kinetic energy & $35 \mathrm{keV}$ \\
Betatron tune & $(1.44,1.44)$ \\
Synchrotron tune & 0.44 \\
rf voltage & $125 \mathrm{~V}$ \\
rf frequency & $46.4 \mathrm{MHz}$ \\
rf harmonics & 2000 \\
Momentum compaction factor & 0.664 \\
\hline \hline
\end{tabular}




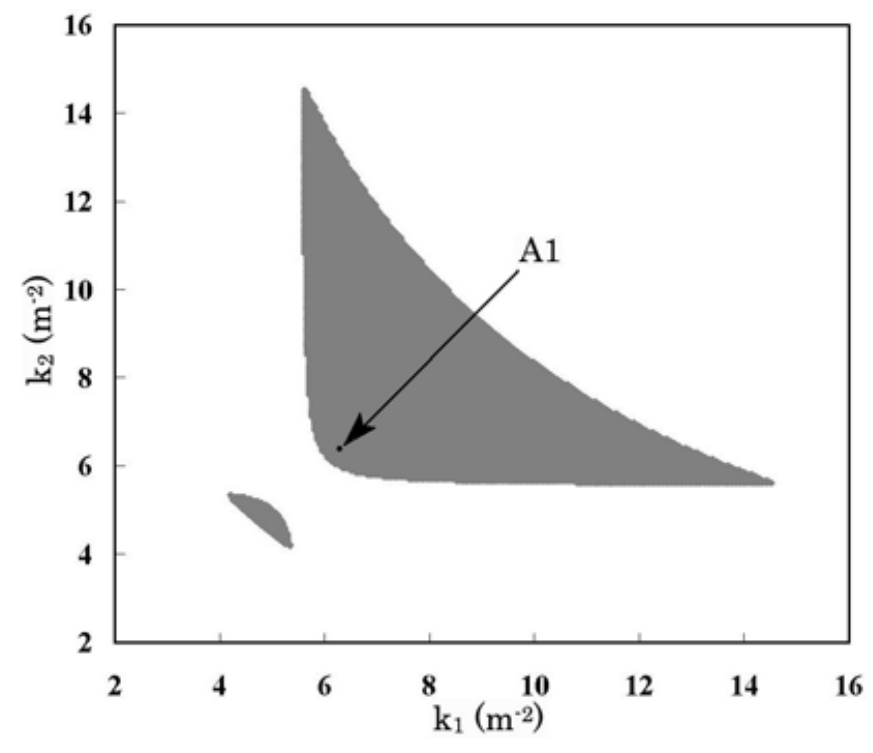

FIG. 6. The stable region of the betatron oscillation. The stable region is plotted on the focusing or defocusing quadrupole plane. The beta functions of the operating point $A 1$ are given in Fig. 7.

dispersion-free condition, the radial focusing has twice the strength of that of the dipole magnet. In S-LSR, the radial focusing of the deflection element is utilized for the horizontal focusing. Thus, the betatron motion of the beam is greatly affected by the radial focusing strength of the deflection element. In the horizontal direction, the strength of the defocusing of the quadrupole magnet has to be increased, in order to compensate for the increase of the radial focusing. Naturally, in connection with it, the focusing strength of the quadrupole magnet in the vertical direction becomes large. Because of such reasons, the betatron tune, especially the vertical tune of the

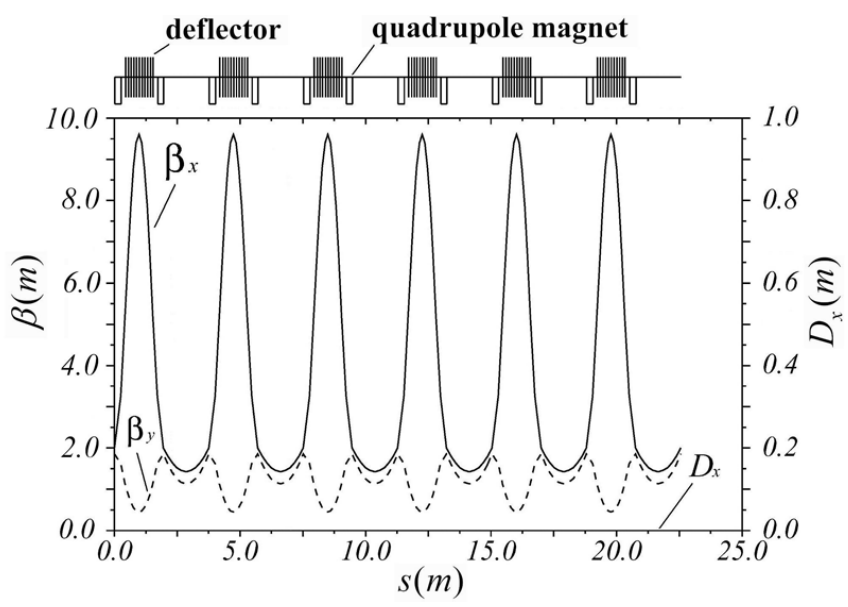

FIG. 7. Beta functions of the operating point $A 1$ of Fig. 6. The tune values are $(1.49,3.49)$. The beta functions are drawn as a function of the position $s$. The positions of the deflectors and quadrupole magnets are shown in the above diagram. $D_{x}$ is the horizontal dispersion function.

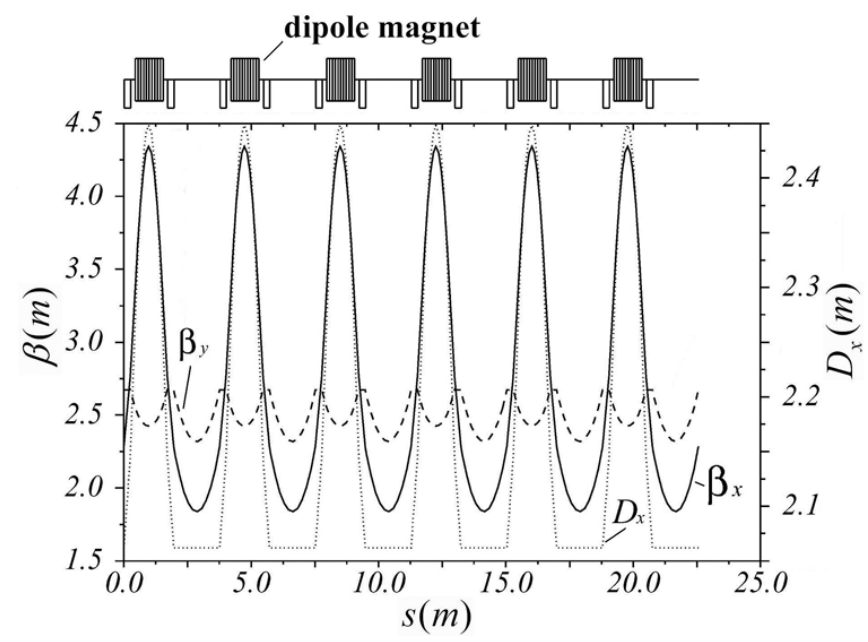

FIG. 8. Beta functions as a function of the position $s$. In this case deflection elements are used as conventional dipole magnets. The betatron tune values are $(1.44,1.44)$.

dispersion-free mode, is increased and, thus, the second criterion for maintaining the crystal beams cannot be satisfied.

The lattice design of S-LSR had been designed to satisfy the two theoretical requirements maintaining the crystalline structure (see Sec. III D) in the magnetic mode [29]. The dispersion-free mode of S-LSR has advantage in the cancellation of the shear heating mechanism, although the second criterion for maintaining the crystal beams is not satisfied. Therefore, in order to achieve a crystal beam, a different form of electric field is required (in order to suppress the vertical tune in S-LSR). In fact, such a form can be found, as will be described in a future publication.

\section{Field error}

A dispersion-free storage ring has never been constructed. Thus, we investigate the influence of the field error of the dispersion suppressor. In the case of S-LSR, the construction errors of the electrostatic deflector will cause larger field error than that of the bending magnet because of its structure, i.e., the gap of the electrostatic deflector is smaller than that of the bending magnet, thus the error of the gap size causes the larger error of the field strength, and the existence of the intermediate electrodes complicates the situation.

Closed orbit distortion (COD) is considered as an influence of the field error. The COD of the dispersion-free mode of S-LSR should be estimated exactly, because the horizontal aperture of the deflectors is only $30 \mathrm{~mm}$. The coefficient $V_{0}$ in the Hamiltonian (10) represents the strength of the bending electric field. Thus, the error of $V_{0}$ will generate the closed orbit distortion. Similarly, the error of the strength of the dipole magnetic field will generate the COD. However, from the field measurement, we found that the field distribution errors of the dipole 
magnets are much smaller compared with electric field, and the individual difference of each magnetic field strength can be corrected by adjusting the current with a bypass circuit.

The main cause of the error of the bending electric field is gap size error of the electrostatic deflector. But, it is difficult to reduce the gap size error less than $0.1 \mathrm{~mm}$ from the circumstances on manufacture. To cope with such a situation, careful adjustment of applied voltage to the main electrodes is planned, taking the real measured gap size into account. In order to realize such adjustment, every main electrode in the six deflection elements is required to be powered by an individual high voltage supply. With the condition that $\Delta V_{0} / V_{0}$ is suppressed to be less than $1.6 \times 10^{-4}$, the COD is expected to be less than $\pm 1 \mathrm{~mm}$, which is thought to be a tolerable size for beam circulation.

\section{Consideration of the nonlinear effect}

We derive the higher-order Hamiltonian of the dispersion suppressor; see the Appendix. From the Hamiltonian of the dispersion suppressor, the Hamiltonian of the dipole magnet with flat field is easily derived by setting the electric field $V_{0}$ to zero. If the higher-order Hamiltonian of the dispersion suppressor is investigated and compared to that of the dipole magnet, one finds the dispersion suppressor inevitably includes nonlinear terms, which are thought to be generated by nonlinear field. The reason is simple: the electric field has radial position dependence (2), thus it includes nonlinear components inevitably. It is expected that the larger nonlinear component of the dispersion suppressor limits the dynamic aperture and causes resonances. The effect of such nonlinear components is evaluated from the experimental result of the KEK electrostatic storage ring [30]. The Hamiltonian of the electrostatic deflector of the electrostatic storage ring is also easily given, if the polarity of the scalar potential is reversed and the vector potential of the magnetic field is eliminated from the Hamiltonian (3) and the equilibrium condition (7). Then, one will find the nonlinear components of the electrostatic deflector has the same formula as that of the dispersion-free deflector.

In the KEK electrostatic storage ring, the measured $1 / e$ lifetimes of stored ions are from $12-20 \mathrm{~s}$. The major factor determining the beam lifetime is interaction with the residual gas, rather than the nonlinear field effect. This result means that the higher-order resonances and the reduction of the dynamic aperture induced by nonlinear field components do not give a large effect to the beam dynamics, in a case considering a vacuum condition and a beam circulation time similar to the KEK ring (if the tune value of the operating point is selected suitably). Therefore, in the case of S-LSR, a similar result is expected.

The bending radius of S-LSR $(1.05 \mathrm{~m})$ is larger than that of the KEK electrostatic storage ring $(0.25 \mathrm{~m})$. According to the higher-order Hamiltonian $\tilde{H}_{2}$ (see the Appendix), the higher-order terms induced by the nonlinear component of the bending electric field are in proportion to $1 / \rho_{0}^{2}$ or $1 / \rho_{0}^{3}$. Therefore, if the bending radius $\rho_{0}$ is increased, the total effect of the nonlinear fields per one turn becomes smaller. Furthermore, real aperture and beam emittance of S-LSR are small originally, thus the reduction of the dynamic aperture may not become the problem. It is known generally that the nonlinear effects become weak, as the beam size and emittance are reduced. Therefore, once a crystalline beam (which is the ultimate low emittance beam) is formed, the nonlinear effects become still smaller, although the nonlinear effects may influence the formation process of the crystal.

\section{CONCLUSIONS}

A method to realize a dispersion-free storage ring is described. The dispersion-free storage ring might be useful for the stabilization of a crystalline beam, because the dynamics of the beam becomes equivalent to that of the linear ion trap. MD simulations based on the Hamiltonian without the shear term were already performed in Ref. [9], and it showed the generation of a 3D crystalline beam, although the beam focusing force of this MD simulation was time independent. A MD simulation including the time-dependent alternating focusing force and higherorder nonlinear effects will be the scope of our further investigation.

This scheme is introduced to the ion cooler ring S-LSR, which is now under construction. In S-LSR, the dispersion suppressor is realized by inserting a cylindrical electrostatic deflector into the small gap of the dipole magnet. The calculation of the beam dynamics has been performed based on the parameters of the deflectors. From the result of the theoretical investigations, we have found that the dispersion-free mode of S-LSR is a stable circulating mode of the beam free from the shear heating mechanism, and provides a lot of capability for beam dynamics study, although the dispersion-free mode of S-LSR has such a restriction as not satisfying the second maintenance condition for beam crystallization. However, this problem can be solved by introducing a different type of electrostatic field. The details of the new type of electric field will be reported in a future publication.

\section{ACKNOWLEDGMENTS}

The work presented here is supported by the Advanced Compact Accelerator Research Project of Ministry of Education, Sports, Culture, Science and Technology. The authors are grateful to all the members of the S-LSR project both in ICR, Kyoto University and National Institute of Radiological Sciences. Their thanks are especially due to fruitful discussions with Dr. K. Noda on the requirement for orbit dispersion with momentum differ- 
ence. They would like to present their thanks also to Dr. J. Wei for his useful theoretical discussions.

\section{APPENDIX: HIGHER-ORDER HAMILTONIAN}

Here, we consider the Hamiltonian of the dispersion suppressor up to the third order. In this case, if the Hamiltonian (3) is expanded and the condition of the equilibrium orbit (7) is substituted, it becomes

$$
\begin{aligned}
\tilde{H}= & \frac{q V_{0}}{\beta_{0}^{2} E_{0}} \frac{x}{\rho_{0}}+\frac{1}{2}\left(1+\frac{q V_{0}}{\beta_{0}^{2} E_{0}}\right)\left(\frac{x}{\rho_{0}}\right)^{2}-\left(1+\frac{x}{\rho_{0}}\right) \frac{\Delta p}{p_{0}} \\
& +\frac{\tilde{p}_{x}^{2}+\tilde{p}_{y}^{2}}{2}-\frac{\tilde{p}_{x}^{2}+\tilde{p}_{y}^{2}}{2} \frac{\Delta p}{p_{0}}+\frac{\tilde{p}_{x}^{2}+\tilde{p}_{y}^{2}}{2} \frac{x}{\rho_{0}} .
\end{aligned}
$$

The momentum $p=\sqrt{\left(p_{t}+q \phi_{D}\right)^{2} / c^{2}-m^{2} c^{2}}$ is expanded up to the power of $\left(\Delta E-q \phi_{D}\right)^{3}$, then the momentum deviation can be written as

$$
\begin{aligned}
\Delta p \approx & \frac{\Delta E-q \phi_{D}}{\beta_{0} c}-\frac{1}{2 p_{0}}\left(\frac{\Delta E-q \phi_{D}}{\beta_{0} c \gamma_{0}}\right)^{2}+\frac{1}{2 p_{0}^{2} \gamma_{0}^{2}} \\
& \times\left(\frac{\Delta E-q \phi_{D}}{\beta_{0} c}\right)^{3} .
\end{aligned}
$$

When Eq. (A2) is inserted into (A1), the Hamiltonian becomes

$$
\tilde{H}=\tilde{H}_{1}+\tilde{H}_{2},
$$

where $\tilde{H}_{1}$ is the Hamiltonian up to the second order and $\tilde{H}_{2}$ is the third-order term:

$$
\begin{aligned}
\tilde{H}_{1}= & -\frac{\Delta E}{\beta_{0}^{2} E_{0}}+\frac{1}{2 \gamma_{0}^{2}}\left(\frac{\Delta E}{\beta_{0}^{2} E_{0}}\right)^{2}+\frac{\tilde{p}_{x}^{2}+\tilde{p}_{y}^{2}}{2}-\frac{\Delta E}{\beta_{0}^{2} E_{0}} \frac{x}{\rho_{0}}(1 \\
& \left.-\frac{q V_{0}}{\gamma_{0}^{2} \beta_{0}^{2} E_{0}}\right)+\frac{1}{2}\left[1+\frac{1}{\gamma_{0}^{2}}\left(\frac{q V_{0}}{\beta_{0}^{2} E_{0}}\right)^{2}\right] \frac{x^{2}}{\rho_{0}^{2}}, \\
\tilde{H}_{2}= & -\frac{1}{2 \gamma_{0}^{2}}\left(\frac{\Delta E}{\beta_{0}^{2} E_{0}}\right)^{3}-\frac{\tilde{p}_{x}^{2}+\tilde{p}_{y}^{2}}{2}\left(\frac{\Delta E}{\beta_{0}^{2} E_{0}}\right)+\frac{\tilde{p}_{x}^{2}+\tilde{p}_{y}^{2}}{2}(1 \\
& \left.-\frac{q V_{0}}{\beta_{0}^{2} E_{0}}\right) \frac{x}{\rho_{0}}+\frac{1}{2 \gamma_{0}^{2}}\left(\frac{\Delta E}{\beta_{0}^{2} E_{0}}\right)^{2} \frac{x}{\rho_{0}}\left(1-3 \cdot \frac{q V_{0}}{\beta_{0}^{2} E_{0}}\right) \\
& +\frac{1}{2 \gamma_{0}^{2}}\left(\frac{\Delta E}{\beta_{0}^{2} E_{0}}\right) \frac{x^{2}}{\rho_{0}^{2}} \frac{q V_{0}}{\beta_{0}^{2} E_{0}}\left(1-3 \cdot \frac{q V_{0}}{\beta_{0}^{2} E_{0}}\right)-\frac{1}{2 \gamma_{0}^{2}} \frac{x^{3}}{\rho_{0}^{3}} \\
& \times\left(\frac{q V_{0}}{\beta_{0}^{2} E_{0}}\right)^{2}\left(1+\frac{q V_{0}}{\beta_{0}^{2} E_{0}}\right)-\frac{1}{3} \frac{x^{3}}{\rho_{0}^{3}}\left(\frac{q V_{0}}{\beta_{0}^{2} E_{0}}\right) .
\end{aligned}
$$

[1] J. J. Bollinger and D. J. Wineland, Phys. Rev. Lett. 53, 348 (1984).

[2] F. Diedrich, E. Peik, J. M. Chen, W. Quint, and H. Walther, Phys. Rev. Lett. 59, 2931 (1987).
[3] M. Drewsen, C. Brondersen, L. Hornkær, J. S. Hangst, and J. P. Schiffer, Phys. Rev. Lett. 81, 2878 (1988).

[4] M. Steck, K. Beckert, H. Eickhoff, B. Franzke, F. Nolden, H. Reich, B. Schlitt, and T. Winkler, Phys. Rev. Lett. 77, 3803 (1996).

[5] H. Danared, A. Källberg, K.-G. Rensfelt, and A. Simonsson, Phys. Rev. Lett. 88, 174801 (2002).

[6] H. Okamoto, K. Okabe, Y. Yuri, D. Möhl, and A.M. Sessler, Phys. Rev. E 69, 066504 (2004).

[7] T. Schätz, U. Schramm, and D. Habs, Nature (London) 412, 717 (2001); U. Schramm, T. Schätz, and D. Habs, Phys. Rev. E 66, 036501 (2002).

[8] U. Schramm, T. Schätz, and D. Habs, Phys. Rev. Lett. 87, 184801 (2001).

[9] A. Rahman and J.P. Schiffer, Phys. Rev. Lett. 57, 1133 (1986).

[10] J. Wei, X.-P. Li, and A.M. Sessler, Phys. Rev. Lett. 73, 3089 (1994).

[11] J. Wei, H. Okamoto, and A. M. Sessler, Phys. Rev. Lett. 80, 2606 (1998).

[12] H. Okamoto and J. Wei, Phys. Rev. E 58, 3817 (1998).

[13] T. Kihara, H. Okamoto, Y. Iwashita, K. Oide, G. Lamanna, and J. Wei, Phys. Rev. E 59, 3594 (1999).

[14] H. Okamoto, Nucl. Instrum. Methods Phys. Res., Sect. A 532, 32 (2004).

[15] W. Henneberg, Ann. Phys. (Berlin) 19, 335 (1934).

[16] W.E. Millet, Phys. Rev. 74, 1058 (1948).

[17] A. Noda, Nucl. Instrum. Methods Phys. Res., Sect. A 532, 150 (2004); A. Noda, H. Fadil, S. Fujimoto, M. Ikegami, Y. Iwashita, S. Nakamura, T. Shirai, M. Tanabe, H. Tongu, K. Matsukado, K. Noda, S. Shibuya, T. Takeuchi, S. Yamada, H, Daido, Y. Kato, T. Tajima, M. Beutelspacher, M. Grieser, and E. Syresin, in Proceedings of the European Particle Accelerator Conference (EPAC), Lucerne, Switzerland, 2004, http:// accelconf.web.cern.ch/accelconf/e04/HTML/

AUTHOR.HTML; A. Noda, in Proceedings of the Workshop on Ion Beam Cooling toward the Crystalline Beam, Kyoto, 2001, edited by A. Noda and T. Shirai (World Scientific, Singapore, 2001), p. 3.

[18] S. Y. Lee, Accelerator Physics (World Scientific, Singapore, 1999), p. 30. The notation of the longitudinal component of vector potential As in this paper coincides with A2 of this reference.

[19] H. Okamoto and S. Machida, Nucl. Instrum. Methods Phys. Res., Sect. A 482, 65 (2002).

[20] R. W. Hasse and J.P. Shiffer, Ann. Phys. (N.Y.) 203, 419 (1990).

[21] The term ground state is used to describe the periodic lowest energy state of the beam in Ref. [10].

[22] The shear term corresponds to the term $\gamma x P_{z}$ in Ref. [10]. There, this term is called "shear."

[23] M. Block, A Drakoudis, H. Leuthner, P. Seibert, and G. Werth, J. Phys. B 33, L375 (2000).

[24] T. Suzuki, Part. Accel. 12, 237 (1982). In order to derive Eq. (20), we used Eq. (15) of this reference.

[25] H. Okamoto, Phys. Rev. E 50, 4982 (1994).

[26] H. Okamoto, A. M. Sessler, and D. Möhl, Phys. Rev. Lett. 72, 3977 (1994). 
[27] M. Ikegami, M. Tanabe, T. Shirai, H. Tongu, K. Noda, M. Grieser, and A. Noda, Nucl. Instrum. Methods Phys. Res., Sect. A 532, 492 (2004).

[28] The transfer matrix calculation was performed by using the code MAD8. The notation of the phase space vector agrees with MAD formalism by easy scaling of the components of the vector. We newly defined the transfer matrix of the dispersion suppressor in MAD. Refer to the following about the calculation method: H. Grote and F. C. Iselin, The MAD Program User's Reference Manual, Report No. CERN/SL/90-13, 1996; F. C. Iselin, The MAD
Program Physical Method Manual, Report No. CERN/SL/ 92, 1994.

[29] T. Shirai, H. Fadil, M. Ikegami, A. Morita, H. Tongu, A. Yamazaki, Y. Iwashita, A. Noda, K. Okabe, Y. Yuri, H. Okamoto, and M. Grieser, in Proceedings of the European Particle Acclerator Conference (EPAC), Paris, 2002 (EPS-IGA and CERN, Geneva, 2002), p. 623.

[30] T. Tanabe, K. Chida, K. Noda, and I. Watanabe, Nucl. Instrum. Methods Phys. Res., Sect. A 482, 595 (2002); T. Tanabe and K. Noda, Nucl. Instrum. Methods Phys. Res., Sect. A 496, 233 (2003). 